\title{
Simulation and optimization of beam losses during continuous transfer extraction at the CERN Proton Synchrotron
}

\author{
Javier Barranco García ${ }^{1,2}$ and Simone Gilardoni ${ }^{1}$ \\ ${ }^{1}$ CERN, Geneva 23, CH-1211, Switzerland \\ ${ }^{2}$ Universitat Politècnica de Catalunya, Campus Diagonal Nord, Edificio B4, C. Jordi Girona, 1-3, 08034 Barcelona, Spain
}

(Received 20 June 2010; published 22 March 2011)

\begin{abstract}
The proton beams used for the fixed target physics at the Super Proton Synchrotron (SPS) are extracted from the Proton Synchrotron (PS) by a multiturn technique called continuous transfer (CT). During the CT extraction, large losses are observed in locations where the machine aperture should be large enough to accommodate the circulating beam. This limits the maximum intensity deliverable due to the induced stray radiation outside the PS tunnel. Scattered particles from the interaction with the electrostatic septum are identified as the possible source of these losses. This article presents a detailed study aiming to understand the origin of losses and propose possible cures. The simulations could reproduce accurately the beam loss pattern measured in real machine operation and determine the beam shaving, intrinsic to the extraction process, as the cause for the unexpected losses. Since these losses are unavoidable, the proposed solution implies a new optics scheme displacing the losses to a region with better shielding. New simulations demonstrate the satisfactory performance of the new extraction optics and its suitability to be implemented in the machine. Finally, beam loss measurements in these new operation conditions confirmed the previous simulation results.
\end{abstract}

DOI: 10.1103/PhysRevSTAB.14.030101

PACS numbers: 29.20.dk, 29.27.Ac

\section{INTRODUCTION}

The Proton Synchrotron (PS) is the oldest element in the CERN accelerator complex, however capable of delivering a wide variety of beams for different purposes [1]. According to the characteristics of the beam the extraction procedure varies. Three different extraction techniques are used in the PS: fast, multiturn or continuous transfer (CT), and slow or resonant extraction.

On a yearly basis, radiation surveys are carried out to monitor radiation levels in the specific areas outside the tunnel where large losses occur (typically injection and extraction regions), in addition to the surroundings of office buildings. During the 2006 survey, two hot areas were identified (Fig. 1): the injection region from PS booster (stations 4 to 5) and building 151 (stations 22 to 23 and 29 to 35, where the first two are located just outside building 151 while the other seven are measured inside [2]). Building 151 is also known as PS bridge because the PS tunnel traverses it, as depicted in Fig. 2. Losses in the injection region can be explained by the injection process itself; however, losses in building 151 are unexpected as there are no aperture limitations in that region.

The radiation surveys do not specify which specific process is causing the losses. An analysis of beam loss monitor (BLM) measurements during all PS cycles

Published by American Physical Society under the terms of the Creative Commons Attribution 3.0 License. Further distribution of this work must maintain attribution to the author(s) and the published article's title, journal citation, and DOI. revealed that only during $\mathrm{CT}$ extraction the beam is lost in that particular region. Figure 3 shows the BLM readings (i.e. cumulative losses) for a complete CERN neutrinos to Gran Sasso $(\mathrm{CNGS})^{1}$ beam cycle. There are three main loss locations after the extraction process starts: first BLMs 5 to 10 , where the saturation of BLM number 9 matches up with the high radiation dose in building 151 that appeared during the 2006 survey (Fig. 1); second, BLM 16 where the magnetic septum to extract the beam towards SPS is located; third, BLMs 30 to 35 where the saturation of BLM 31 indicates the location of the electrostatic septum used to slice the beam during the CT extraction. Studies were launched [4] to explain why these losses arose, considering that there are no aperture limitations in the PS ring in that area. The outcome of these studies pointed out particles scattered by the electrostatic septum blade as the source of the losses. This study confirms the assumptions regarding the beam loss mechanism and proposes and implements a solution to reduce the losses in building 151 .

\section{PRINCIPLE OF THE CT EXTRACTION}

The PS ring is composed of 100 combined-function magnets arranged in a focusing-defocusing-defocusingfocusing lattice and interleaved by 100 straight sections (SS, numbered from SS00 to SS99). All the machine

\footnotetext{
${ }^{1}$ The CNGS beam is delivered to the SPS for neutrino production as a part of the CERN neutrinos to Gran Sasso experiment [3].
} 


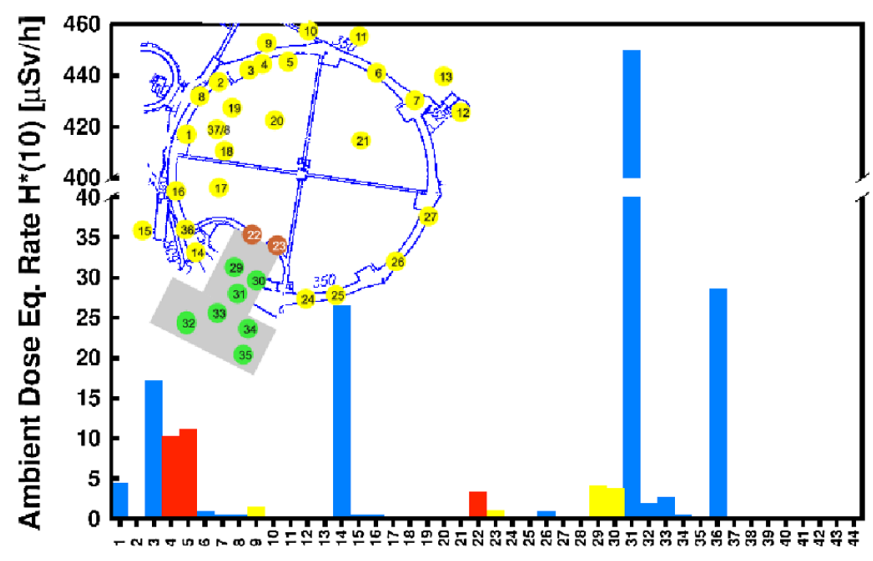

FIG. 1. Result of the 2006 PS radiation survey in $\mu \mathrm{Sv} / \mathrm{h}$ for a circulating intensity of $0.8 \times 10^{13} \mathrm{p}^{+} / \mathrm{sec}$ [2]. Measurements were taken from 44 different stations spread all along the PS tunnel (top left). The color code in the histogram represents the ratio between the measured dose and the guideline value specified in [15]. In blue, stations where the dose is below $30 \%$ of the guideline value; in yellow, stations where the measured dose is between 30 and $100 \%$; in red, where the measured dose is above $100 \%$. The stations measuring in building 151 are $22-23$ (brown) and 29-35 (green).

elements are named with reference to the straight section where they are located.

The CT extraction is used to fill the SPS in two consecutive PS runs (circumference SPS $=11 \times$ circumference $_{\mathrm{PS}}$ ). All elements used in this process are displayed in Fig. 4.

When the $14 \mathrm{GeV} / c$ flattop is reached, the PS horizontal tune is set to $Q_{x}=6.25$ which provides a phase advance per turn of $90^{\circ}$, while the vertical one remains fixed at $Q_{y}=6.3$. A few milliseconds before the extraction, two slow bumps, BSW31 and BSW16, are powered. The first one approaches the beam to the electrostatic septum in SS31 (SEH31), whereas the second does it to the magnetic septum in SS16 (SMH16). Simultaneously, two quadrupoles located in SS25 and SS05, which form the so-called

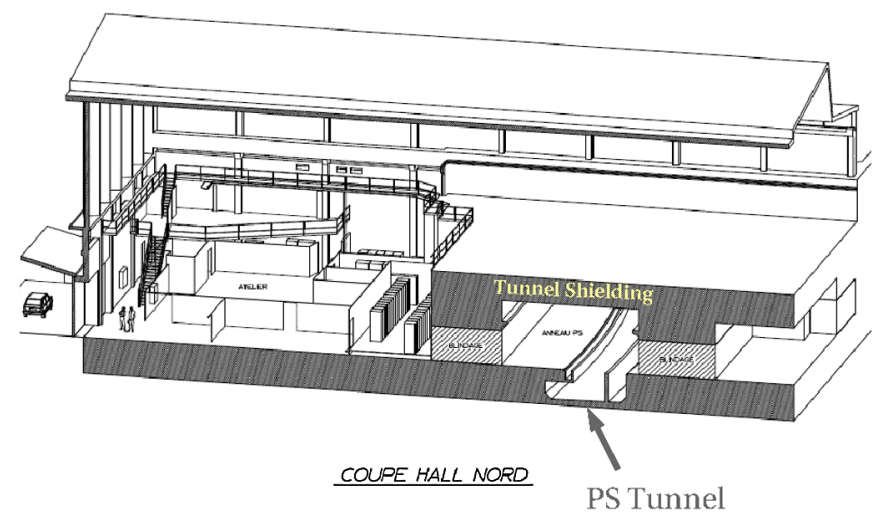

FIG. 2. Drawing of the PS bridge building 151. The tunnel PS traverses it (bottom right) being not possible to increase the shielding in that region.

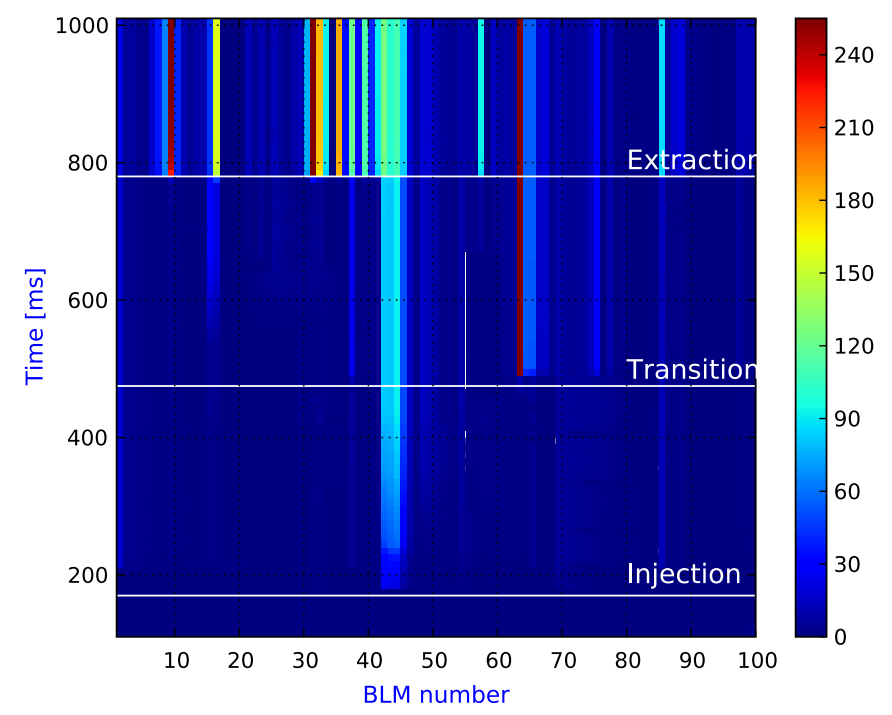

FIG. 3. BLMs integrated measurement for the CNGS beam. The BLM signal is divided into 255 bits, beyond that value the BLM saturates. The timings for injection, transition crossing, and extraction are shown. Losses below the PS bridge are measured by BLMs 5-9.

QKE16 circuit, are used to alter the optics between them (Fig. 5), in such a way that large $\beta_{x}$ and almost zero $D_{x}$ values are obtained in the SEH31 location while the optics remain unchanged in the SMH16 location. These changes increase the beam size and reduce the energy dependence in SEH31, minimizing the losses at the septum while keeping the beam size small during the extraction in SMH16. When the beam is close enough to SEH31 and the first losses appear, two fast magnets with rise time much shorter than the revolution period, namely, BFA21 and BFA09, will be used to adjust the relative position between beam and septum blade on a turn-by-turn basis, to obtain an equal intensity extraction per turn (Fig. 6). The portion of the beam above the blade will receive an additional kick that along with BSW16 will drive the particles above SMH16 and will extract them to the transfer line towards

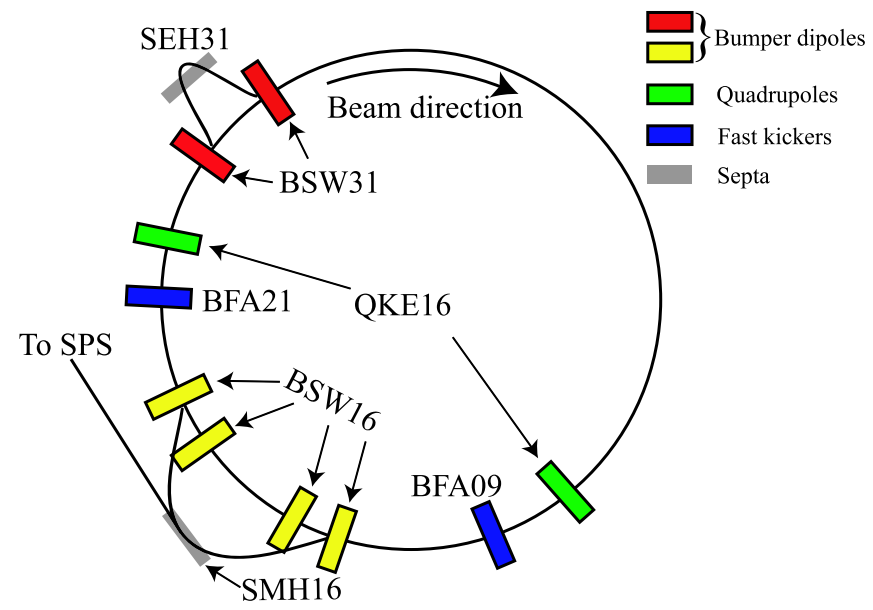

FIG. 4. Layout of all elements used during the CT extraction. 


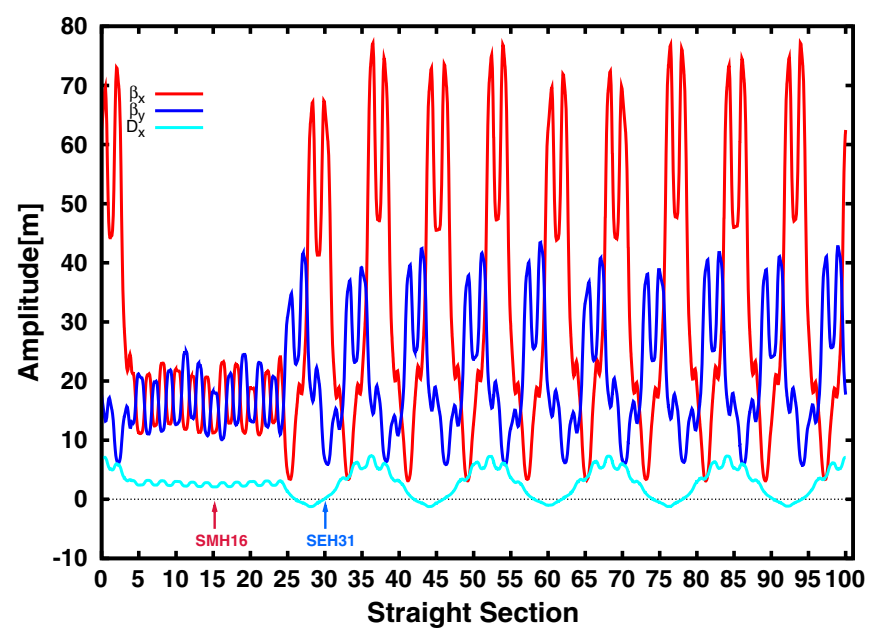

FIG. 5. PS optics distorted by the QKE16. The region at small horizontal and vertical $\beta$ is between SS05 and SS25. The locations of SMH16 and SEH31 are pointed out.

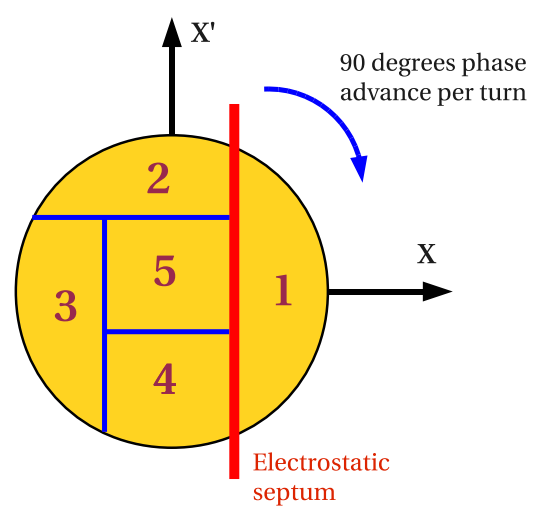

FIG. 6. Five slices in normalized phase space. $Q_{x}=6.25$ corresponds to $90^{\circ}$ phase advance per turn. As the $\mathrm{C}_{\mathrm{SPS}}=$ $11 \times \mathrm{C}_{\mathrm{PS}}$, two consecutive PS extraction of five slices each are needed to fill the SPS completely.

the SPS. The strengths of the fast kickers are increased during the five turns to assure the equal population between the different slices. A typical time structure of the BFAs strength is shown in Fig. 7. The total kick is divided into a constant strength or pedestal (red), and an increasing one or staircase (blue) to refine the adjustment. As described in [5], it is not possible to generate slices with same emittances and same intensities at the same time: the condition of equal intensity is the most suitable to reduce beam instabilities in the SPS.

The typical five turns long spill structure measured by the current transformer in the transfer line from PS to SPS is shown in Fig. 8. By integrating the signal the extraction efficiency for each turn is obtained and shown in Table I. According to this, the two main parameters taken into

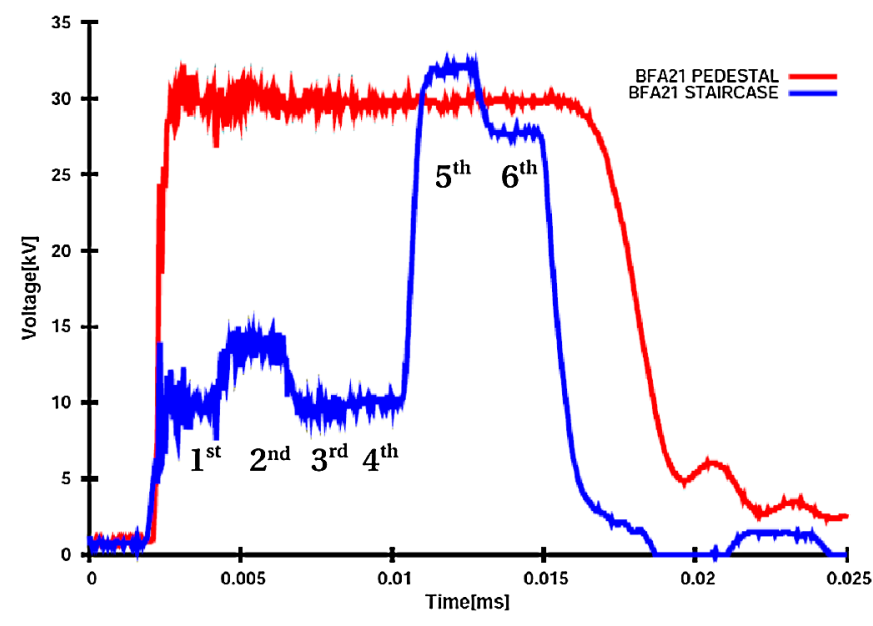

FIG. 7. BFA21 kicker strength in $\mathrm{kV}$ during the five extraction turns. The pulse length corresponds to six machine turns. The last part (6th) turn is used to empty the machine. During the fifth turn the remaining central part of the beam is placed above the septum to be extracted and a higher voltage is needed.

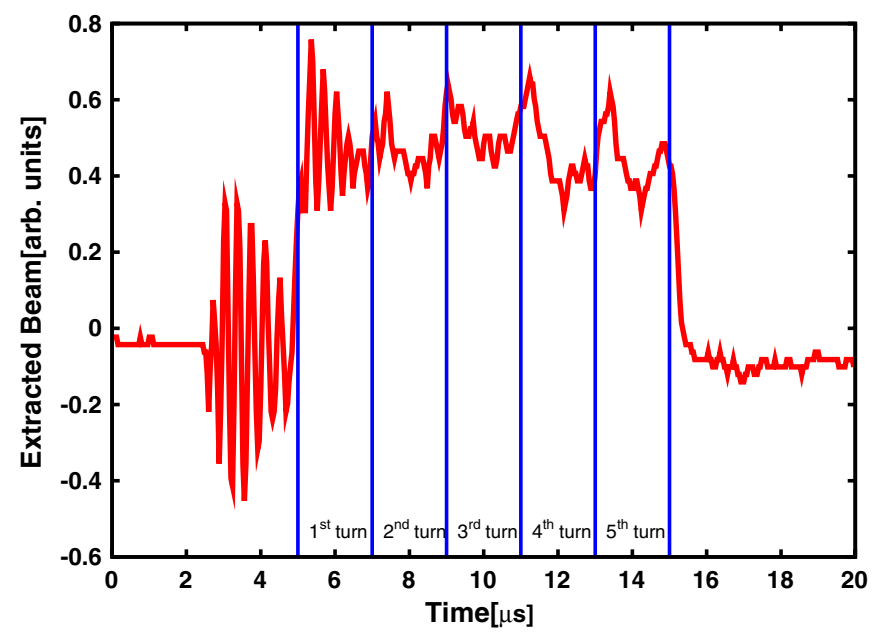

FIG. 8. Five turn spill structure measured by a current transformer in the transfer line between the PS and the SPS. Some noise on the reading is observed before the extraction and during the first turn.

account in order to validate the simulations are an equal intensity extraction $(\sim 20 \%$ per slice $)$ and an overall average efficiency of the process of $\sim 93 \%$. The simulations described in the following sections include tracking

TABLE I. Extraction efficiency measurements for each of the five turns, with an overall average efficiency of $\sim 93 \%$.

\begin{tabular}{lc}
\hline \hline Turn & $\begin{array}{c}\text { Beam } \\
\text { Extracted (\%) }\end{array}$ \\
\hline 1st & 18.1 \\
2nd & 18.5 \\
3rd & 20.5 \\
4th & 18.1 \\
5th & 17.8 \\
\hline \hline
\end{tabular}


through the PS lattice with the fast bumps changing turn by turn, particles interaction with the septum blade, and finally trajectory comparison with the PS aperture model to determine the loss locations.

\section{A. Optics of the CT extraction}

During operation the radial position of the beam, thus the energy, is adjusted to match the injection energy of the SPS. A typical radial displacement implemented in the PS for this reason is of the order of $\Delta x=-3 \mathrm{~mm}$ giving a momentum error of about $\delta p / p=-0.001$. In addition, there is a peak to peak value of $\Delta x=8 \mathrm{~mm}$ in the closed orbit measured just before the extraction starts. Figure 9 shows the MADX [6] orbit model used in the simulations which includes the real closed orbit measured by the BPMs and the radial displacement.

Particle tracking around the ring is performed with a modified version of the SIXTRACK code [7] based on the MADX model provided. In order to simulate the change in the closed orbit during the five turns extraction two dipoles, varying strengths turn by turn, are included in the code. The kick provided by the fast bumps is calculated by

$$
\begin{aligned}
\theta_{\mathrm{SS} 21} & =-U_{\mathrm{S}, \mathrm{N}} \frac{\int B d l_{\mathrm{st}}}{U_{\mathrm{max}, \mathrm{st}}} \frac{1}{B \rho}-U_{\mathrm{P} 21} \frac{\int B d l_{\mathrm{ped}}}{U_{\mathrm{max}, \mathrm{ped}}} \frac{1}{B \rho} \\
\theta_{\mathrm{SS} 9} & =-U_{\mathrm{S}, \mathrm{N}} \frac{\int B d l_{\mathrm{st}}}{U_{\mathrm{max}, \mathrm{st}}} \frac{1}{B \rho}-U_{\mathrm{P} 9} \frac{\int B d l_{\mathrm{ped}}}{U_{\mathrm{max}, \mathrm{ped}}} \frac{1}{B \rho}
\end{aligned}
$$

with $U_{\mathrm{S}, \mathrm{N}}$ the voltage of the staircase for the turn $N, U_{\mathrm{max}, \mathrm{st}}$ maximum voltage of the staircase, $U_{\mathrm{P} 21}$ and $U_{\mathrm{P} 9}$ voltage for fast bumpers in 21 and $9, U_{\text {max,ped }}$ maximum voltage of the pedestal, $B \rho$ the magnetic rigidity, and $\int B d l$ the integrated magnetic field for the maximum voltages $U_{\text {max,ped }}$ and $U_{\text {max,st }}$. In real operation the fast bumps are

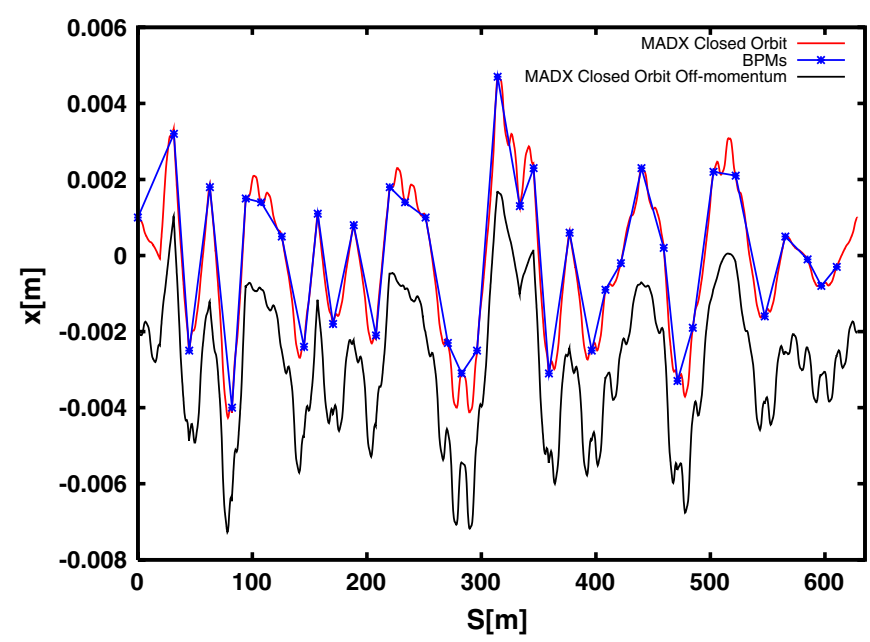

FIG. 9. PS closed orbit simulated and measured before the pulsing of the slow bumps.

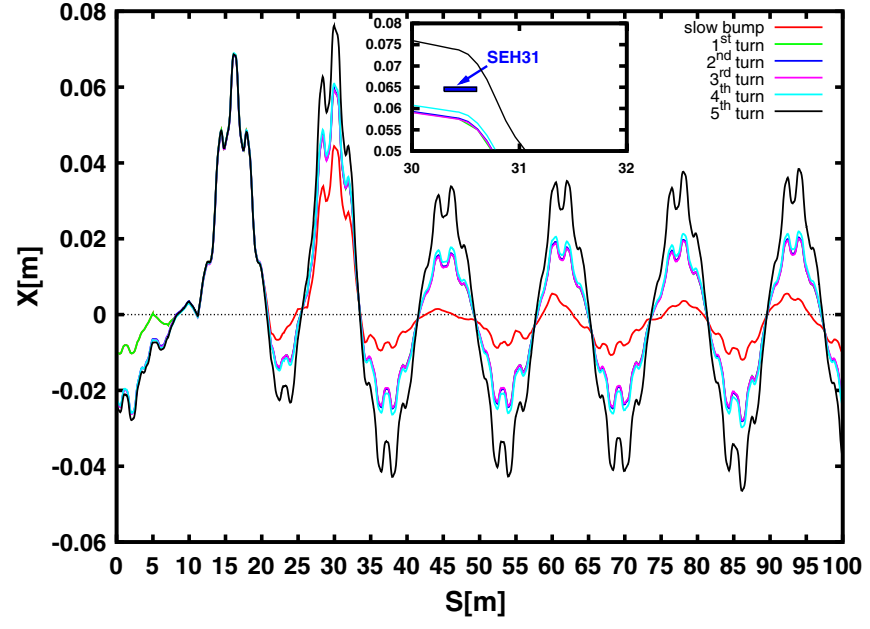

FIG. 10. Simulated closed orbit in the tracking code and zoom of the position between septum and closed orbit. The necessary fast-bump values for an equal intensity extraction turn by turn are calculated considering a bi-Gaussian beam distribution. The zoom indicates the position of SEH31 with respect to the closed orbits for each turn.

adjusted manually until the equal extraction is achieved. Normalizing the coordinates, $X=\frac{x}{\sqrt{\beta}}$ and $X^{\prime}=$ $\frac{\alpha}{\sqrt{\beta}} x+\sqrt{\beta} x^{\prime}$, and integrating over the distribution (biGaussian in phase space) to obtain $1 / 5$ of intensity in each slice as shown in Fig. 6, the relative position between beam and septum blade can be calculated. From these values, the needed closed orbit per each extraction turn can be calculated and with Eq. (1) the coefficients for the pedestal and staircase are then adjusted. Figure 10 shows the simulated closed orbits for the slow bumps and the five consecutive extraction turns. While the closed orbit peak in SS16 is kept constant, the one in SS31 increases according to the values calculated before. The zoomed area in Fig. 10 shows the relative position between septum blade and closed orbit during the five turn process. Only in the fifth turn the closed orbit goes above the septum as the central part of beam has to be extracted in the last turn. Table II

TABLE II. Parameters used in the simulations to achieve the equal intensity extraction. The second column is the relative position of the beam and septum blade for a bi-Gaussian beam. The third and fourth columns show BFA kicks in mrad calculated from the needed closed orbit. The last column shows extracted efficiency for each of the five turns.

\begin{tabular}{lcccc}
\hline \hline Turn & $\begin{array}{c}\text { Relative } \\
\text { Position }\end{array}$ & $\begin{array}{c}\text { Kick } \\
\text { BFA21 }\end{array}$ & $\begin{array}{c}\text { Kick } \\
\text { BFA9 }\end{array}$ & $\begin{array}{c}\text { Beam } \\
\text { Extracted [\%] }\end{array}$ \\
\hline 1st & 0.84 & -0.5225 & -0.5070 & 20.0 \\
2nd & 0.67 & -0.4793 & -0.4638 & 18.5 \\
3rd & 0.62 & -0.4953 & -0.4798 & 19.2 \\
4th & 0.32 & -0.5864 & -0.5709 & 19.0 \\
5th & $\cdots$ & -1.0236 & -1.0081 & 19.5 \\
\hline \hline
\end{tabular}




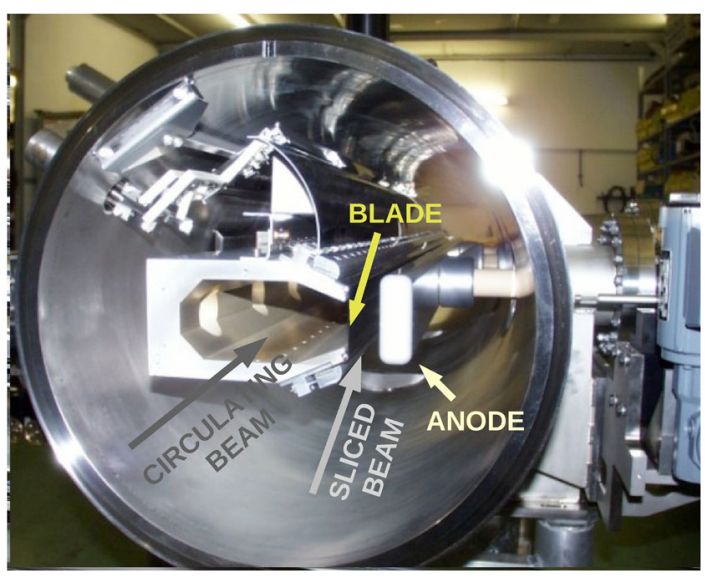

FIG. 11. Electrostatic septum 31. The circulating beam passes in the left vacuum pipe, whereas the electrical field is present at the right of the septum blade.

summarizes the parameters for each turn used in the simulations to set up the equal intensity extraction. These values are included in the SIXTRACK model of the PS lattice.

\section{B. Model of the electrostatic septum}

The interaction of particles with the septum blade is included in the simulations with the K2 code [8]. The electrostatic septum in SS31 is composed of a $1.8 \mathrm{~m}$ long molybdenum blade creating an electrostatic field with the anode as shown in Fig. 11. The vacuum pipe is earthed so the circulating beam is not affected. The main septum parameters used in the simulation are presented in Table III.

The kick given by an electrostatic septum is calculated with [9]

$$
\theta=\arctan \left(\frac{e E_{0} l}{p c \beta}\right)
$$

where $e$ is the electronic charge, $E_{0}$ the electric field gradient, $l$ the electrode length, $p$ the particle momentum, $c$ the speed of light, and finally, $\beta$ the relativistic normalized speed. For the values in Table III, the maximum kick from SEH31 is $\theta_{\max }=0.86 \mathrm{mrad}$. The $\mathrm{K} 2$ model used in the simulations takes into account all possible cases when a

TABLE III. Main SEH31 septum parameters.

\begin{tabular}{lcc}
\hline \hline Parameter & Value & Unit \\
\hline Electrode length & 1850 & $\mathrm{~mm}$ \\
Septum length & 2293 & $\mathrm{~mm}$ \\
Septum half gap & 65 & $\mathrm{~mm}$ \\
Blade nominal thickness & 0.1 & $\mathrm{~mm}$ \\
Operative gap width & 27 & $\mathrm{~mm}$ \\
Angle with respect to beam & -0.0006 & $\mathrm{rad}$ \\
Voltage (DC) & 170 & $\mathrm{kV}$ \\
\hline \hline
\end{tabular}

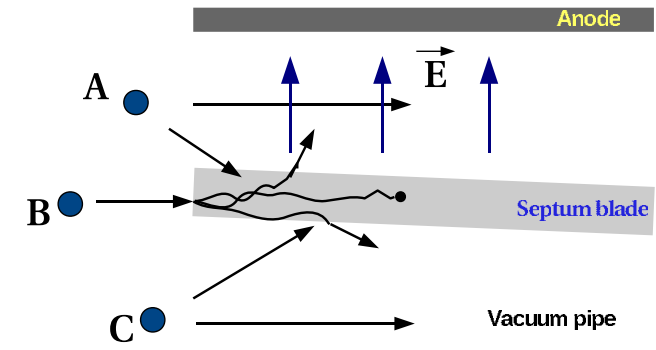

FIG. 12. Sketch of all possible cases when a particle reaches the septum.

particle reaches the septum (Fig. 12). If a particle arrives above the septum (case A in Fig. 12), it can either traverse the whole electrostatic field receiving the maximum kick or, if its divergence is negative, it might end up hitting the septum blade. On the other hand, if a particle arrives below the septum (case $\mathrm{C}$ in Fig. 12), it can either continue circulating freely in the vacuum chamber or, if its divergence is positive, it could hit the septum blade at some point. Finally, a particle could hit the septum in the front (case B in Fig. 12). In all the cases that the particle traverses a certain length of material, it will undergo all the processes included in K2, i.e., multiple Coulomb scattering, ionization energy loss, and nuclear interactions [10]. In the event of a particle outscattered into the electrostatic field region, the kick applied is proportional to the remaining length traversed inside it.

\section{PS aperture model}

An accurate aperture model is essential to reproduce the loss pattern measured in real operation by the BLMs. Because of this, special effort was put in order to measure and establish it, based on dimensions from mechanical drawings for each element and measurements made in situ [11]. Figure 13 shows the horizontal (bottom) and vertical (top) aperture model used to compare with particle trajectories to determine the loss locations.

\section{BEAM LOSS MONITOR SYSTEM}

In order to be able to correlate simulations with measurements, it is necessary to understand how losses are measured in the PS. The PS beam loss monitor system is composed by 100 aluminum cathode electron multiplier (ACEM) detectors [12], mounted on top of the main magnets. Because of the position chosen in the past (the system dates back to the middle 1980s) and the large variation of the signal with the primary proton energy, it is practically impossible to correlate the amount of protons lost in the machine with the signal recorded by the BLMs, even if all the BLMs are calibrated every year to assure a uniform response for a given loss [13]. Moreover, most of the losses 

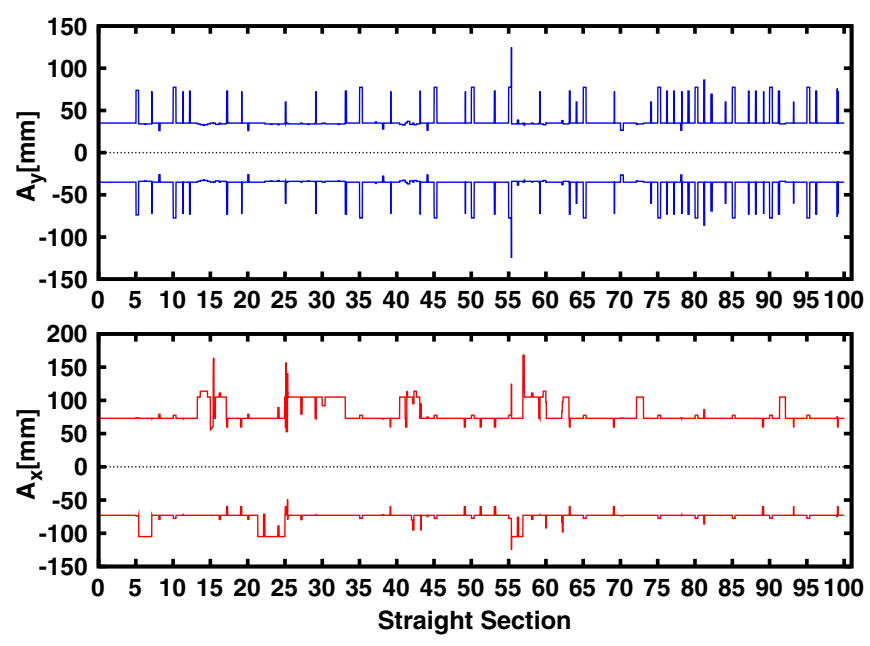

FIG. 13. PS aperture model in mm. Vertical (top), horizontal (bottom).

in the PS are in the horizontal plane, whereas the BLMs are mounted on top of the bulky iron yoke of the main magnets just after each SS as shown in Fig. 14. In addition, the BLMs are installed on different sides of the main magnets, sometimes facing the inside (as in Fig. 14) of the ring, sometimes outside, following the pattern of the four magnet types which compose the PS lattice. According to their location, they cover a different solid angle directly affecting the measurements. For these reasons, the BLM system is used during operation to detect malfunctioning of the machine, and not as a real protection system, obtained by comparing online a given loss pattern with the reference one.

The pattern of the losses observed with the system can tell something about the region where the losses occur,

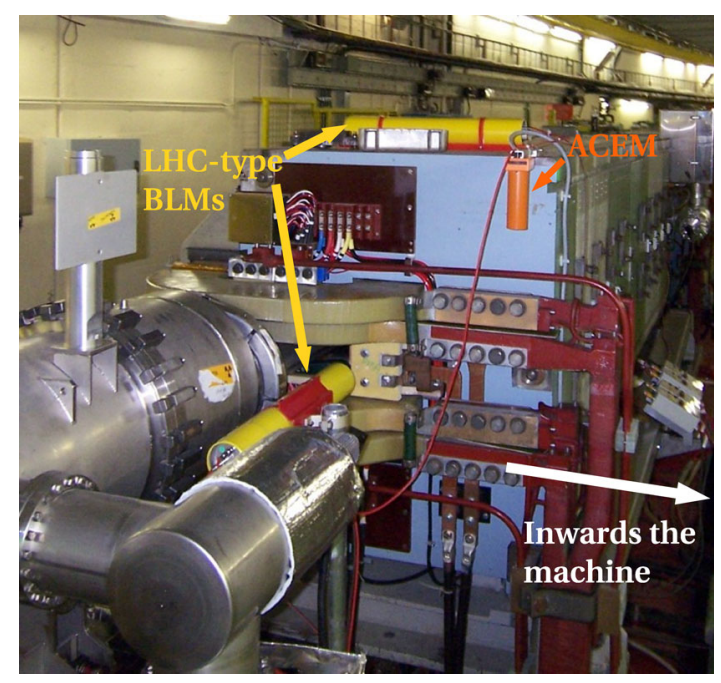

FIG. 14. ACEM detector mounted on the main magnet unit 16. The yellow cylinders on top of the magnet and parallel to the extraction magnetic septum are new BLMs-LHC type under tests. whereas it is not possible, for example, to deduce the ratio of beam lost between two different sections of the machine. It is not even possible to deduce if the loss is produced in one of the main magnets or in the straight sections, as the BLM is mounted at the entrance of the magnet unit. The aim of the simulations presented in the following section is to reproduce qualitatively the observed loss pattern but not to quantify the beam loss detected by the BLMs. This would require a detailed simulation of the secondary shower developing in a large fraction of the main magnets, and goes beyond the purpose of this study.

\section{SIMULATION RESULTS}

The average extraction efficiency achieved during machine operation is $\eta=93.0 \%$ with approximately equal intensity for the different beam slices as presented in Table I, in comparison with simulations which give an overall extraction efficiency of $\eta=96.3 \%$ with an intensity per slice quoted in Table II. This discrepancy can be explained from the fact that in the simulations the real alignment of the septum with the rest of the machine elements considering all of them centered with respect to the closed orbit. In addition, simulations have been performed with the nominal width of the septum; however, the impact of the beam in the septum and the energy deposited could have lead to a deformation of the blade increasing the width. A larger impacting surface can certainly lead to a decrease in the extraction efficiency. The average extraction efficiency evaluated for different alignments and septum widths is presented in Table IV. More realistic scenarios reduce the simulated extraction efficiency to values in good agreement with the measurements during machine operation.

Figure 15 (top) compares beam loss histograms from measurements and simulations considering the nominal parameters, showing a very good qualitative agreement as all the hot locations were found in the latter. Within the $\sim 4 \%$ of losses, $95 \%$ are found to be caused by the scattering in the septum blade. Specifically this is the reason for losses in SS05-09, where the quadrupole located in SS05 (as a part of the QKE16), defocuses the scattered particles and cause the losses. The maximum energy loss simulated for the particles lost in the SS05-10 region is $0.5 \%$ which gives a dispersive contribution to the closed

TABLE IV. Extraction efficiency measurements (\%) simulated for different relative angles between beam and septum blade and different septum widths.

\begin{tabular}{|c|c|c|c|}
\hline Angle [rad] Width $[\mathrm{mm}]$ & 0.1 & 0.2 & 0.3 \\
\hline-0.0002 & 96.1 & 94.5 & 93.4 \\
\hline-0.0006 & 96.3 & 95.3 & 94.2 \\
\hline-0.001 & 95.0 & 94.0 & 92.6 \\
\hline
\end{tabular}



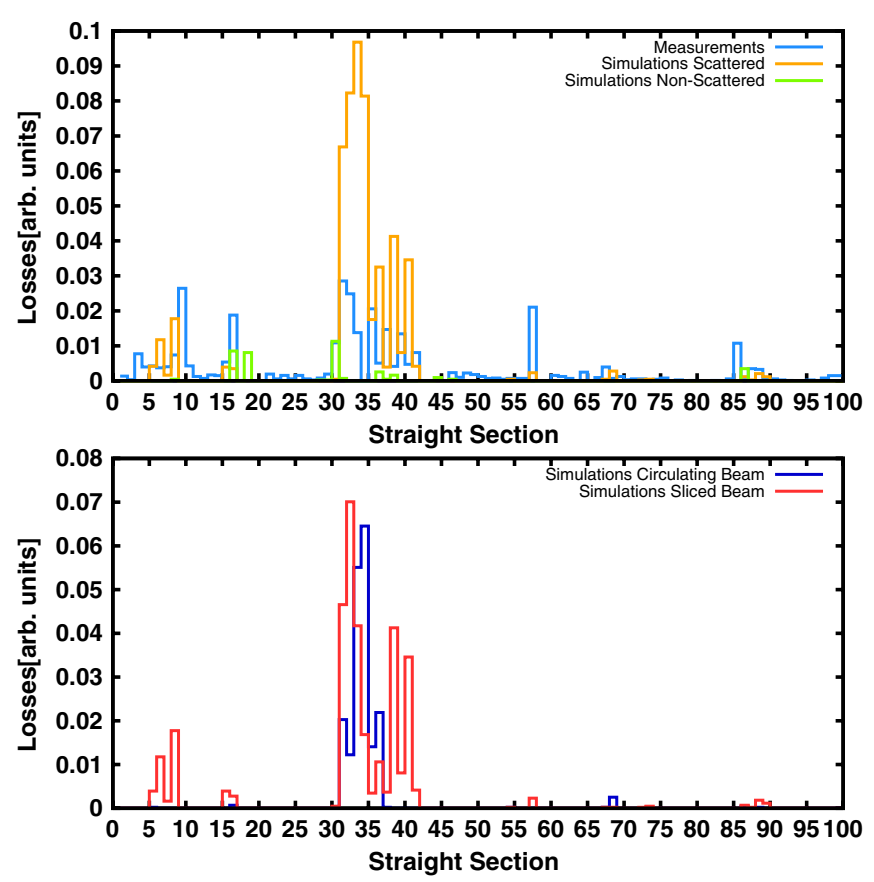

FIG. 15. Beam loss distribution for normal CT operation. Top, BLM measurements (blue) are compared with simulations (green, lost particles nonscattered, and orange, particles scattered by the blade). Bottom, simulation results for scattered particles differentiating into sliced particles (red), i.e., outscattered into the field region, and circulating particles (blue), i.e., outscattered into the vacuum pipe.

orbit of $15 \mathrm{~mm}$ still far from the available aperture confirming the kick provided by the quadrupole SS05 as the main reason for the losses. The other $5 \%$ of losses are particles which have been not scattered by SEH31 but due to a wrong closed orbit are either not kicked properly in SEH31 or extracted in SMH16. However, the particles can be either outscattered towards the anode (sliced beam) thus receiving an additional kick, or towards the center of the vacuum chamber (circulating beam) as shown in Fig. 12. Figure 15 (bottom) compares simulated losses for the two cases, showing that one-third of the particles lost were scattered towards the vacuum chamber and lost in the sections immediately after the septum while the rest were outscattered into the field region spreading more the losses and producing the ones in SS05-09 after being deflected by a quadrupole in SS05. These three results (overall efficiency, turn-by-turn efficiency, and beam loss distribution) confirm the accuracy of the model used for simulations. However, as already mentioned in Sec. III, there are certain issues that prevent from a one-to-one comparison between simulations and real measurements when analyzing the results.

Losses in sections SS05 to SS09 are clearly identified, but in relative terms the loss peaks are smaller in the simulations than in the measurements. This is probably because the simulations do not take into account secondary showers from the particles hitting the machine aperture,

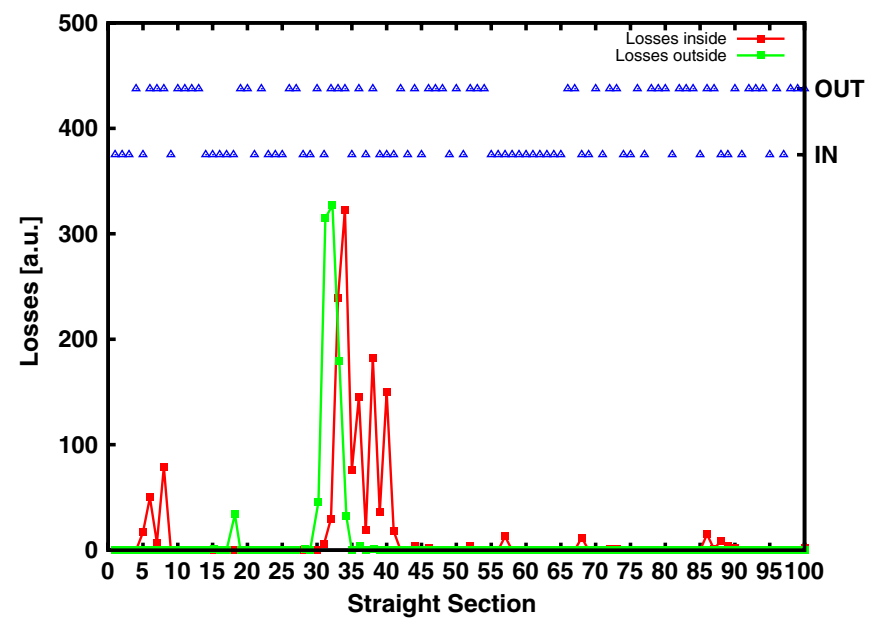

FIG. 16. Beam loss pattern simulation differentiating losses occurred inwards (red) and outwards (green) the machine. In addition, BLMs positions are displayed indicating whether they were installed inside or outside the vacuum chamber. There is a discrepancy in the BLM location and where the losses occurred in SS05-09. BLM in SS09 is located inwards the machine which explains the signal saturation due to the secondary particles produced in SS08.

counting only direct losses. Another significant difference appears in section 57, where the magnetic septum for slow extraction (SMH57) is placed. Those losses come probably again, from secondary particles produced in the septum and measured by the BLM in that location.

The BLMs are mounted, as mentioned before, at the end of each straight section inside or outside depending on the magnet, while in the simulations all particles are grouped and counted considering the whole section producing which can lead to some inconsistency between measurements and simulations.

In Fig. 16 it can be seen that most of the losses occur inwards the machine while in the septum (SS16 and SS31) areas occur outwards, which is consistent with the closed orbit in Fig. 10. It is important to highlight that the BLM in SS09 is located inwards and, thus, measures correctly. That would explain why it is the only one that saturates in that area. Dose measurements on contact done in several points along SS06 to SS10 [14] show that higher doses are found inwards (Fig. 17) in agreement with the simulations performed. The maximum dose appears in SS08 but, as mentioned before, in this case the BLM is placed outwards which could explain why it is not reflected in the BLM measurement. Once more the secondary particles produced by losses in SS08 seem the most probable cause for the saturation of BLM in SS09.

\section{New optics scheme for loss displacement}

Previous simulations confirm particle interaction with the septum blade as the source of losses in SS05 to SS10, making them unavoidable unless the extraction process is 


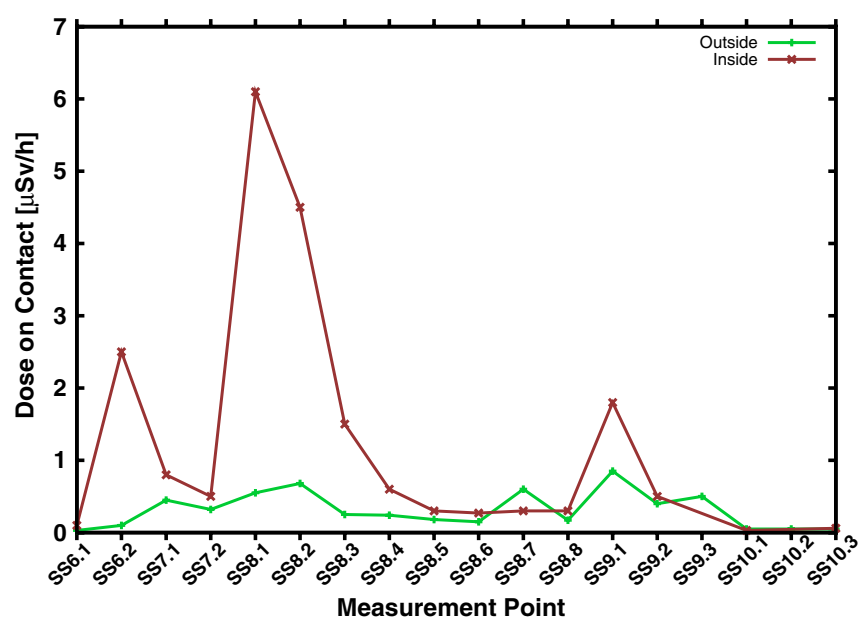

FIG. 17. Dose measurements on contact in several points along SS06 to SS10. In red, doses measured towards inside the ring; in green, towards outside. Losses occur mainly inwards the machine in consistency with simulations in Fig. 16.

changed [5]. In the meantime, the only viable solution to reduce the radiological impact of the extraction losses is to displace them in a better-shielded part of the machine, where the tunnel radiation shield thickness is larger. This can be done by installing a new quadrupole $4 \pi$ in phase advance upstream the quadrupole in SS05 and removing the latter. Then the new extraction optics would be unchanged in the location of the electrostatic and magnetic septa, as shown in Fig. 18. The quadrupole defocusing the scattered particles producing the losses would be now in SS73, bringing the losses into the SS73 to SS78 region. Simulations for this new configuration show a clear displacement of losses from building 151 to the SS73 area as shown in Fig. 19 (top). These losses, as predicted, are scattered particles into the sliced beam (Fig. 19, bottom).

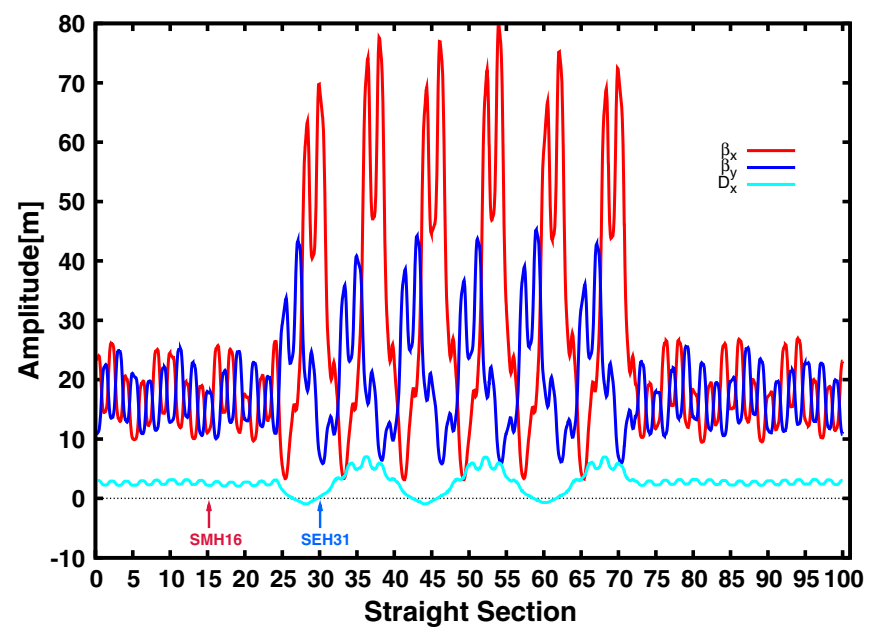

FIG. 18. CT extraction optics for the new QKE16 formed now by quadrupoles in SS25 and SS73. The locations of SMH16 and SEH31 are indicated.
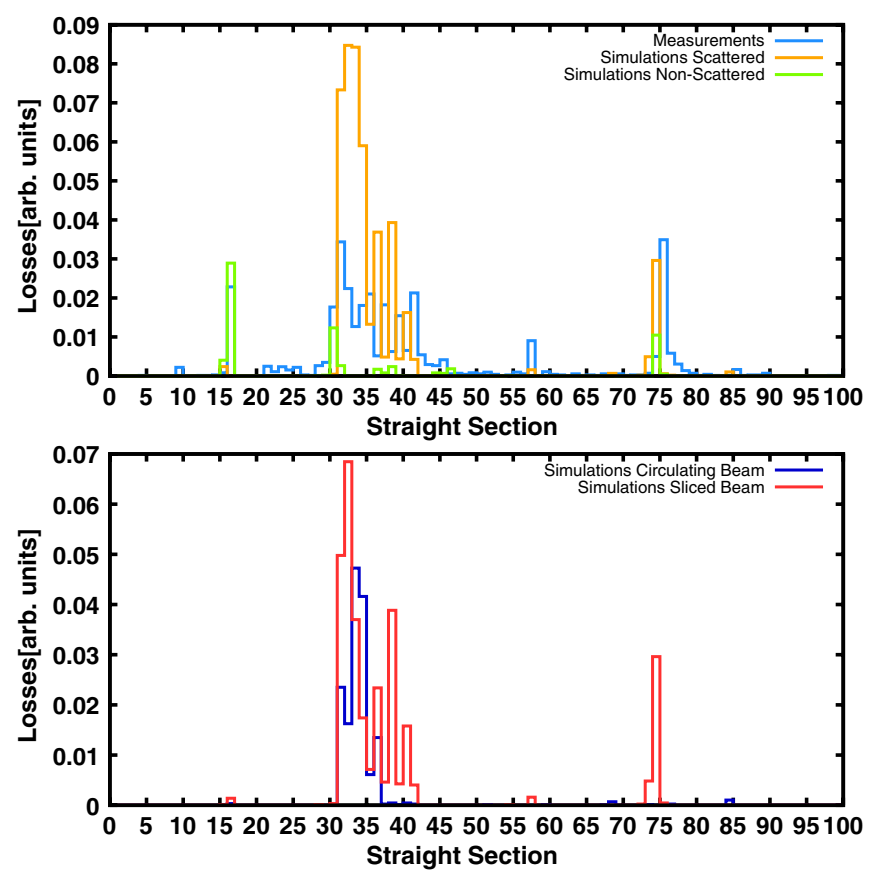

FIG. 19. Beam loss distribution for new CT optics scheme (color legend as in Fig. 15). Beam losses have been displaced from SS05-09 to the SS73 area.

This solution was implemented during the 2007 PS run obtaining the results expected from the simulations. Figure 20 shows the BLMs measurements where the ones in the PS bridge measure almost zero losses, but the one in SS75 saturates; nevertheless the shielding around this region should decrease the dose outside the tunnel. Though a mismatch of one section in SS74 between simulations and measurements can be seen, due to the location of BLM at the entrance of the main magnet that corresponds to the

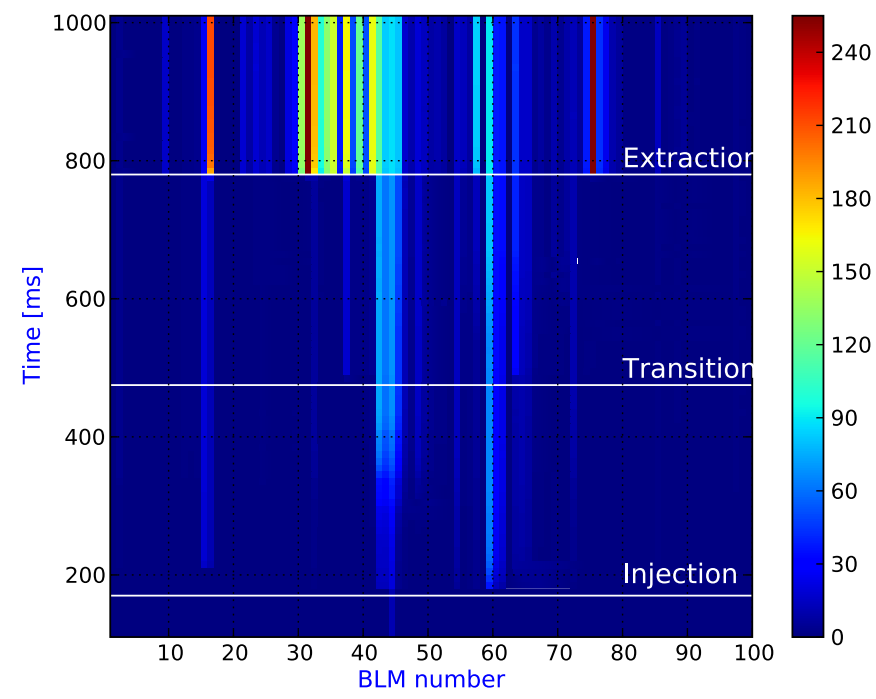

FIG. 20. BLM integrated measurement for the CNGS beam with the new optics scheme. Losses in SS05-09 have been displaced to a better-shielded region in SS75. 


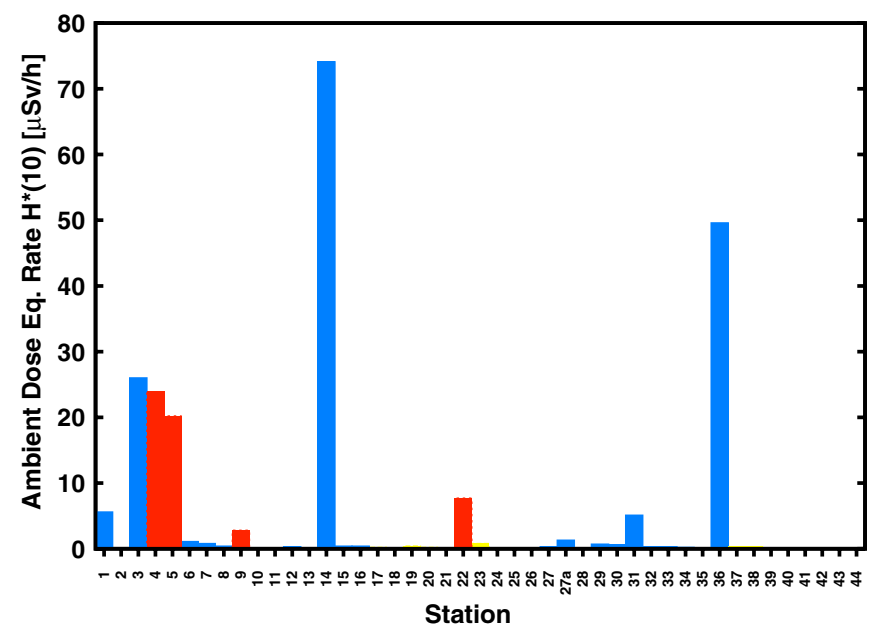

FIG. 21. PS 2007 radiation survey in $\mu \mathrm{Sv} / \mathrm{h}$ [2]. The color code is as in Fig. 1. The dose in stations 29 and 30 now fulfills the value guidelines.

middle of the straight section in the simulations, it is possible that losses occurring at the end of SS74 are measured forward in SS75.

A new radiation survey, similar to the one carried out in 2006, showed that actually the irradiation outside the tunnel has been decreased in the region of the experimental building, whereas a practically negligible increase was observed outside the tunnel near the zone on top of SS75 (station 27a), where most of the losses were concentrated after the quadrupole displacement. The results of the new radiation survey are shown in Fig. 21, where the dose in the inside of the experimental building (stations 29 and 30) was reduced below the guideline values.

\section{CONCLUSIONS}

The present study succeeded to explain the beam loss mechanism producing high radiation doses in the surroundings of the CERN PS building 151 observed during the continuous transfer extraction. The detailed model built for the simulations included five turn tracking with time varying fast kickers, interaction of particles with septum, and loss location by comparison with the PS aperture model. The parameters used to validate the simulations were overall extraction efficiency, turn-by-turn efficiency, and beam loss distributions. However, several issues were mentioned to be taken into account when comparing measurements and simulations. As these losses are unavoidable, a new optics is proposed to displace them to a better-shielded area. Simulations confirmed the expected results leading to an implementation during the 2007 PS run. A radiation survey in 2007 showed a great reduction in the radiation dose in building 151, changing its classification to supervised radiation area. High radiation doses in stations 22 and 23 are planned to be solved by increasing the shielding in that particular area.

\section{ACKNOWLEDGMENTS}

The authors would like to thank G. Arduini, M. Delrieux, M. Giovannozzi, E. Métral, G. Métral, Y. Papaphilippou, and R. Steerenberg for all the useful discussions, M. Widorski, I. Floret, and the other CERN/RP colleagues for providing the data of the radiation survey, $\mathrm{O}$. Berrig and G. Robert-Demolaize for developing and adapting the PS aperture model used in the simulations, R. Tomás for his help adapting the SIXTRACK code, and the members of the CERN TE/ABT/SE section for the help, expertise, and patience during the loss pattern studies.

[1] http://op-webtools.web.cern.ch/op-webtools/dokuwiki/ doku.php?id=cps-beams.

[2] M. Widorski, Accelerator Performance Committee Meeting (APC), 2007.

[3] K. Elsener et al., CERN AC Note No. (2000-03).

[4] S. Gilardoni, Accelerator Performance Committee Meeting (APC), 2006.

[5] M. Giovannozzi et al., Report No. CERN-2006-01.

[6] http://mad.web.cern.ch/mad/.

[7] G. Robert-Demolaize et al., Report No. CERN-AB-2005033.

[8] T. Trenkler and J.B. Jeanneret, Report No. SL/Note 94-105 (AP).

[9] B. Goddard, in Handbook of Accelerator Physics and Engineering, edited by A.W. Chao and M. Tigner (World Scientific, Singapore, 1999), pp. 431-434.

[10] N. Catalan-Lasheras, Report No. CERN-THESIS-2000019.

[11] http://cern-accelerators-optics.web.cern.ch/cern-acceleratorsoptics/PSoptics/defaultPS.htm.

[12] V. Agoritsas et al., Nucl. Instrum. Methods Phys. Res., Sect. A 247, 44 (1986).

[13] V. Prieto, Report No. CERN PS/BD/Note 2001-006, in French.

[14] I. Floret (private communication).

[15] CERN Safety Code F, Protection Against Ionizing Radiation. Radiation Safety Manual. 